\title{
Life threatening experiences as proximal risk factors for suicide attempt: a case-control study
}

\author{
Alejandro Porras-Segovia, Miguel de la Cruz-Barrales, María Luisa Barrigón.
}

Fundación Jiménez Díaz, Madrid

\section{Introduction}

Suicide is a public health priority worldwide. Each year, over 800,000 people take their own life, and about 20 times more attempt to do so. Suicide is a complex phenomenon with not-completely-elucidated etiopathogenic factors. Life Threatening Events are a known risk factor for suicidal behavior, and may play an important role as proximal factors that shortly precede the suicidal act. However, few studies have investigated the dynamics of suicidal risk factors and how they influence the different phases of the suicidal trajectory. Our aim is to explore the proximal association between LTEs and suicide attempts through a case-control study.

\section{Methods}

Cases were adult patients attended by the psychiatrist at the Emergency Room after having committed a suicide attempt. Controls were adult patients attended by the psychiatrist for any other reason. All patients gave written informed consent to participate in the study. Our main independent variable was having experienced a Life Threatening Event in the last six months, which was assessed using the List of threatening experiences Brugha et al. (Brugha \& Cragg, 1990). Other independent variables explored were sex, age, work status, marital status, and DSM-IVbased clinical diagnosis.

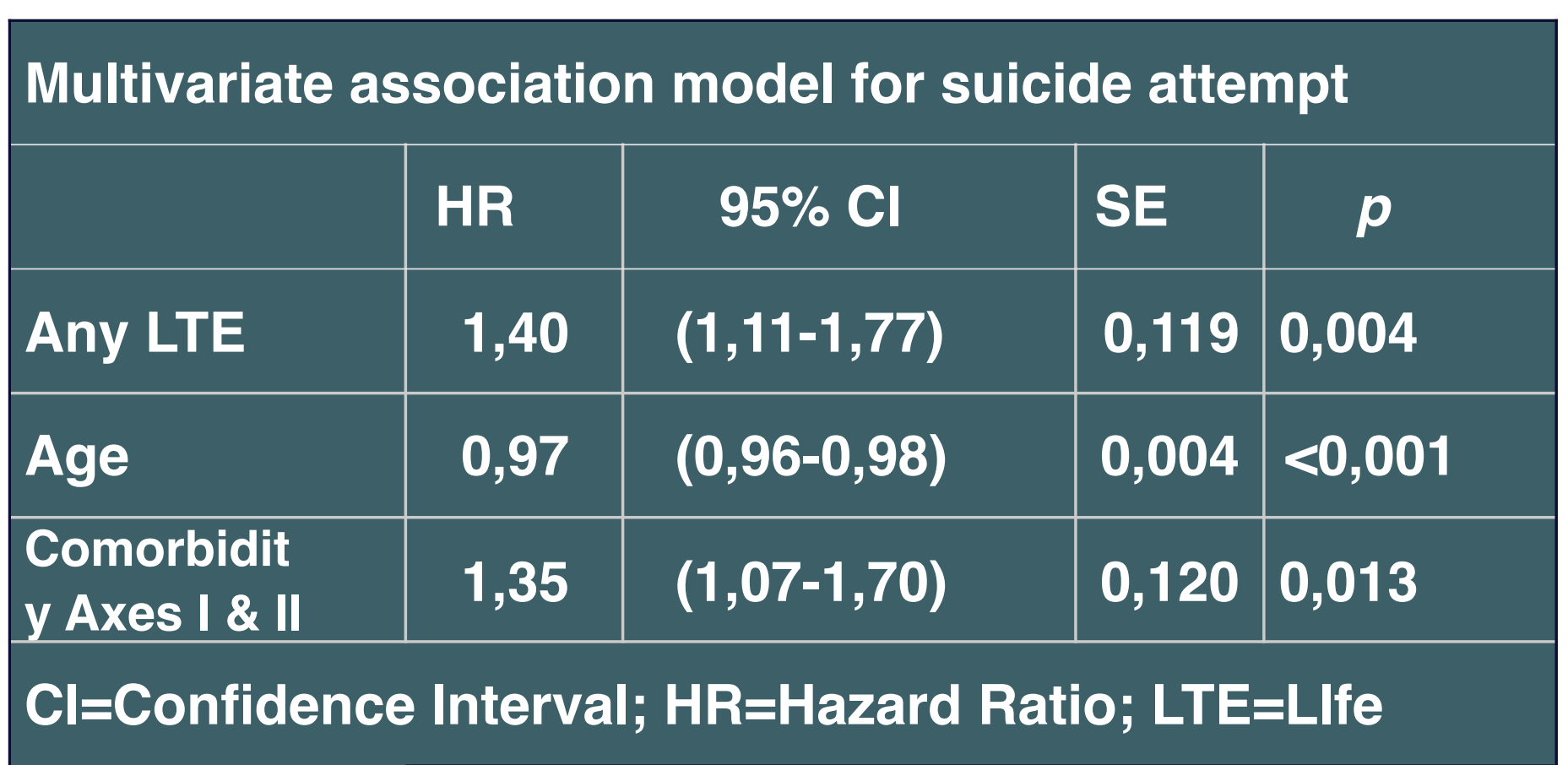

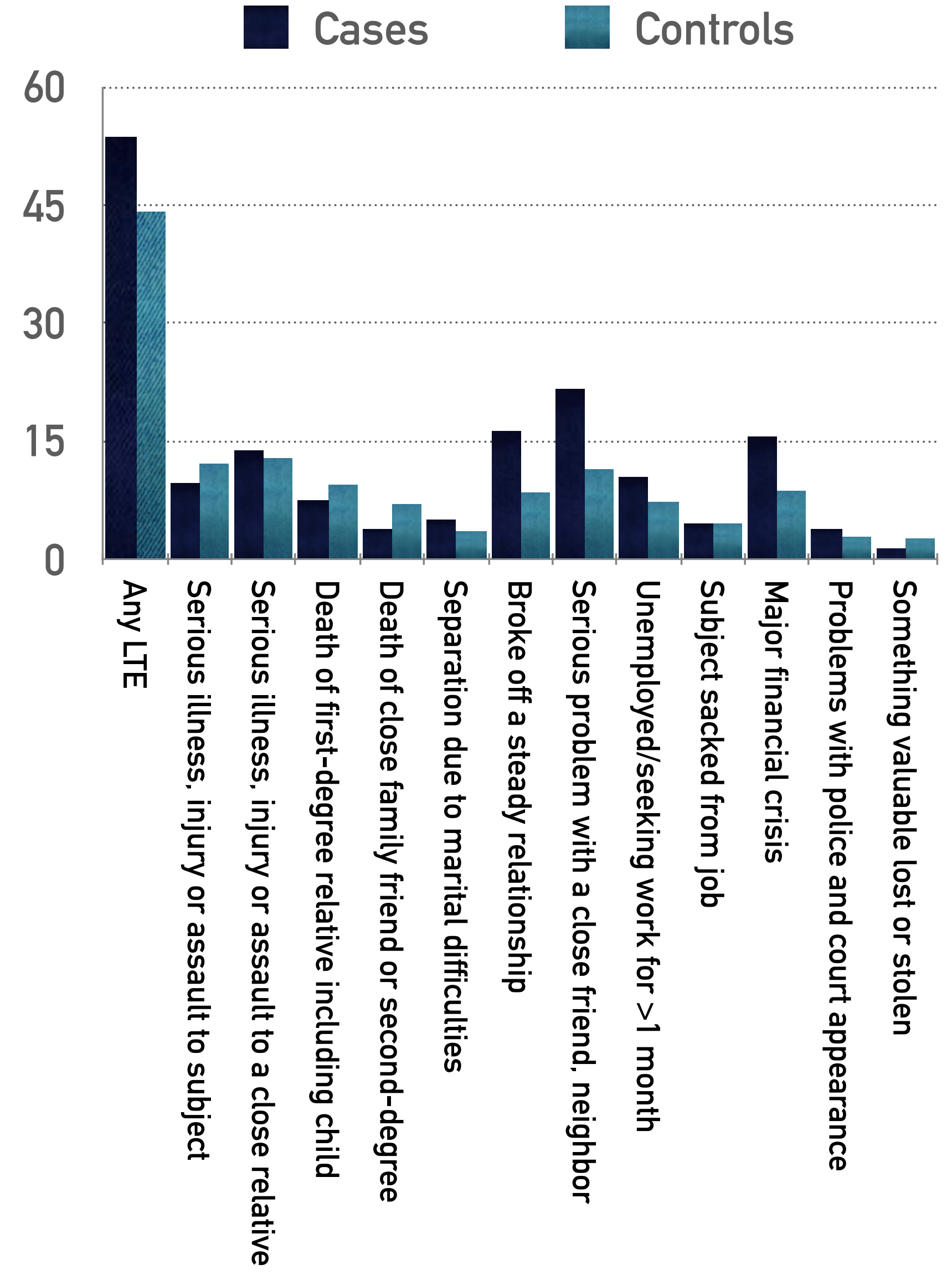

\begin{abstract}
Results
Having experienced any LTE was significantly associated with committing a suicide attempt. Regarding the specific LTEs, that with a statistically significant positive association was having broken off a steady relationship. Three variables were significantly and independently associated with suicide attempts in the multivariate association model: any LTEs, comorbidity between Axis I \& II, and age.
\end{abstract}

\section{Conclusions}

Suffering a LTE significantly increases the risk for suicide in the next 6 months, acting as a proximal factor in the pathway to suicide. Breaking up a relationship emerges as the most powerful of LTE in contrast with more apparently traumatic experiences, such as the loss of a loved one. This highlight the importance of impulsivity and anger as prominent feelings behind suicidal behaviour.

As showed in several previous studies, personality disorders are strong risk factors for suicide. The frequent difficulties with personal relationships and intimacy experienced by people with personality disorder may increase the impact of relationshiprelated LTE as a trigger of suicidal behaviour. 